\title{
Hyperon-hyperon interactions and properties of neutron star matter
}

\author{
I. Vidaña, A. Polls, and A. Ramos \\ Departament d'Estructura i Constituents de la Matèria, Universitat de Barcelona, E-08028 Barcelona, Spain \\ L. Engvik and M. Hjorth-Jensen \\ Department of Physics, University of Oslo, N-0316 Oslo, Norway
}

(Received 11 April 2000; published 27 July 2000)

\begin{abstract}
We present results from Brueckner-Hartree-Fock calculations for $\beta$-stable neutron star matter with nucleonic and hyperonic degrees of freedom, employing the most recent parametrizations of the baryon-baryon interaction of the Nijmegen group. It is found that the only strange baryons emerging in $\beta$-stable matter up to total baryonic densities of $1.2 \mathrm{fm}^{-3}$ are $\Sigma^{-}$and $\Lambda$. The corresponding equations of state are then used to compute properties of neutron stars such as masses and radii.
\end{abstract}

PACS number(s): 13.75.Ev, 21.30.-x, 21.65.+f, 26.60.+c

\section{INTRODUCTION}

The physics of compact objects such as neutron stars offers an intriguing interplay between nuclear processes and astrophysical observables. Neutron stars exhibit conditions far from those encountered on earth; typically, expected densities $\rho$ of a neutron star interior are of the order of $10^{3}$ or more times the "neutron drip" density $\approx 4 \times 10^{11} \mathrm{~g} / \mathrm{cm}^{3}$, where nuclei begin to dissolve and merge together. Thus, the determination of an equation of state (EOS) for dense matter is essential to calculations of neutron star properties. The EOS determines properties such as the mass range, the massradius relationship, the crust thickness, and the cooling rate. The same EOS is also crucial in calculating the energy released in a supernova explosion.

At densities near to the saturation density of nuclear matter $\left(\rho_{0}=0.16 \mathrm{fm}^{-3}\right)$, we expect the matter to be composed of mainly neutrons, protons, and electrons in $\beta$ equilibrium, since neutrinos have on average a mean free path larger than the radius of the neutron star. The equilibrium conditions can then be summarized as

$$
\mu_{n}=\mu_{p}+\mu_{e}, \quad \rho_{p}=\rho_{e},
$$

where $\mu_{i}$ and $\rho_{i}$ refer to the chemical potential and density of particle species $i$, respectively. At the saturation density of nuclear matter $\rho_{0}$, the electron chemical potential is of the order $\sim 100 \mathrm{MeV}$. Once the rest mass of the muon is exceeded, it becomes energetically favorable for an electron at the top of the $e^{-}$Fermi surface to decay into a $\mu^{-}$. A Fermi sea of degenerate negative muons starts then to develop and, consequently, the charge balance needs to be changed according to $\rho_{p}=\rho_{e}+\rho_{\mu}$ as well as requiring that $\mu_{e}=\mu_{\mu}$.

As the density increases, new hadronic degrees of freedom may appear in addition to neutrons and protons. One such degree of freedom is hyperons, baryons with a strangeness content. Contrary to terrestrial conditions where hyperons are unstable and decay into nucleons through the weak interaction, the equilibrium conditions in neutron stars can make the inverse process happen, so that the formation of hyperons becomes energetically favorable. As soon as the chemical potential of the neutron becomes sufficiently large, energetic neutrons can decay via weak strangeness nonconserving interactions into $\Lambda$ hyperons leading to a $\Lambda$ Fermi sea with $\mu_{\Lambda}=\mu_{n}$. However, one expects $\Sigma^{-}$to appear via

$$
e^{-}+n \rightarrow \Sigma^{-}+\nu_{e},
$$

at lower densities than the $\Lambda$, even though $\Sigma^{-}$is more massive. The negatively charged hyperons appear in the ground state of matter when their masses equal $\mu_{e}+\mu_{n}$, while the neutral hyperon $\Lambda$ appears when its mass equals $\mu_{n}$. Since the electron chemical potential in matter is larger than the mass difference $m_{\Sigma^{-}}-m_{\Lambda}=81.76 \mathrm{MeV}, \Sigma^{-}$will appear at lower densities than $\Lambda$. For matter with hyperons as well the chemical equilibrium conditions become

$$
\begin{gathered}
\mu_{\Xi^{-}}=\mu_{\Sigma^{-}}=\mu_{n}+\mu_{e}, \\
\mu_{\Lambda}=\mu_{\Xi^{0}}=\mu_{\Sigma^{0}}=\mu_{n}, \\
\mu_{\Sigma^{+}}=\mu_{p}=\mu_{n}-\mu_{e} .
\end{gathered}
$$

Hyperonic degrees of freedom have been considered by several authors, mainly within the framework of relativistic mean field models [1-3] or parametrized effective interactions [4], see also Balberg et al. [5] for a recent update. Realistic hyperon-nucleon interactions were employed by Schulze et al. [6], in a many-body calculation in order to study the onset of hyperon formation in neutron star matter. In a recent work [7], they extend their work to study the properties of neutron stars with hyperons, paying special attention to the role played by three-body nucleon forces. All these works show that hyperons appear at densities of the order of $\sim 2 \rho_{0}$.

In Refs. [6,7] the hyperon-hyperon interaction was not included. However, it is clear that as soon as the $\Sigma^{-}$hyperon appears, one needs to consider the interaction between hyperon pairs since it will influence the single-particle energy of hyperons, hence affecting the equilibrium conditions from that density on and the density where other hyperons (e.g., the $\Lambda$ ) appear. The aim of this work is thus to present results of many-body calculations for $\beta$-stable neutron star matter with hyperonic degrees of freedom, employing interactions which also account for strangeness $S<-1$. To achieve this 
goal, our many-body scheme starts with the most recent parametrization of the free baryon-baryon potentials for the complete baryon octet as defined by Stoks and Rijken in Ref. [8]. This entails a microscopic description of matter starting from realistic nucleon-nucleon, hyperon-nucleon, and hyperon-hyperon interactions. In a recent work [9] we have developed a formalism for microscopic Brueckner-type calculations of dense nuclear matter that includes all types of baryon-baryon interactions and allows to treat any asymmetry on the fractions of the different species $\left(n, p, \Lambda, \Sigma^{-}, \Sigma^{0}, \Sigma^{+}, \Xi^{-}\right.$, and $\left.\Xi^{0}\right)$.

Here we extend the calculations of Ref. [9] to studies of $\beta$-stable neutron star matter. A brief summary of the formalism discussed in Ref. [9] is presented in Sec. II. Our results are shown in Sec. III. In Sec. III A we discuss the equation of state and the composition of $\beta$-stable matter with strangeness, using various nucleonic contributions to the EOS. Based on the composition of matter we discuss in Sec. III B the possible neutron star structures. Our conclusions are given in Sec. IV.

\section{FORMALISM}

Our many-body scheme starts with the most recent parametrization of the free baryon-baryon potentials for the complete baryon octet as defined by Stoks and Rijken in Ref. [8]. This potential model, which aims at describing all interaction channels with strangeness from $S=0$ to $S=-4$, is based on SU(3) extensions of the Nijmegen potential models [10] for the $S=0$ and $S=-1$ channels, which are fitted to the available body of experimental data and constrain all free parameters in the model. In our discussion we employ the interaction version NSC97E of Ref. [8], since this model, together with the model NSC97F of Ref. [8], results in the best predictions for hypernuclear observables [10]. For a discussion of other interaction models, see Refs. [8,11].

With a given interaction model, the next step is to introduce effects from the nuclear medium. Here we will construct the so-called $G$ matrix, which takes into account shortrange correlations for all strangeness sectors, and solve the equations for the single-particle energies of the various baryons self-consistently. The $G$ matrix is formally given by

$$
\begin{aligned}
&\left\langle B_{1} B_{2}|G(\omega)| B_{3} B_{4}\right\rangle \\
&=\left\langle B_{1} B_{2}|V| B_{3} B_{4}\right\rangle+\sum_{B_{5} B_{6}}\left\langle B_{1} B_{2}|V| B_{5} B_{6}\right\rangle \\
& \times \frac{Q}{\omega-E_{B_{5}}-E_{B_{6}}+\imath \eta}\left\langle B_{5} B_{6}|G(\omega)| B_{3} B_{4}\right\rangle .
\end{aligned}
$$

Here $B_{i}$ represents all possible baryons $n, p, \Lambda, \Sigma^{-}, \Sigma^{0}$, $\Sigma^{+}, \Xi^{-}$, and $\Xi^{0}$ and their quantum numbers such as spin, isospin, strangeness, linear momenta, and orbital momenta. $Q$ is the Pauli operator which allows only intermediate states $B_{5} B_{6}$ compatible with the Pauli principle, and the energy variable $\omega$ is the starting energy defined by the singleparticle energies of the incoming external particles $B_{3} B_{4}$. The $G$ matrix is solved using relative and center-of-mass

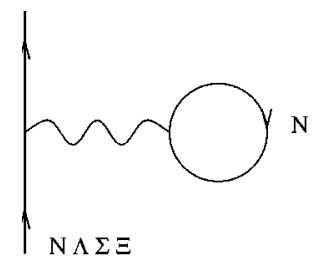

a)

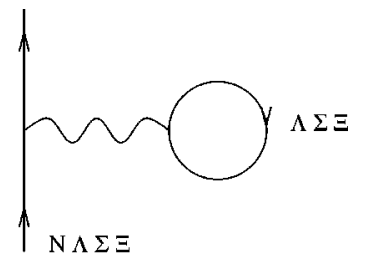

b)
FIG. 1. Goldstone diagrams for the single-particle potential $U_{B}$. (a) represents the contribution from nucleons only as hole states while (b) includes only hyperons as hole states. The wavy line represents the $G$ matrix.

coordinates, see, e.g., Refs. [9,11] for computational details. The single-particle energies are given by

$$
E_{B_{i}}=T_{B_{i}}+U_{B_{i}}+M_{B_{i}}
$$

where $T_{B_{i}}$ is the kinetic energy and $M_{B_{i}}$ the mass of baryon $B_{i}$. We note that the $G$ matrix, Eq. (4), has been solved using the standard prescription (i.e., $E_{B}=T_{B}+M_{B}$ ) for the intermediate states $B_{5} B_{6}$. The single-particle potential $U_{B_{i}}$ is defined by

$$
U_{B_{i}}=\operatorname{Re} \sum_{B_{j} \leqslant F_{j}}\left\langle B_{i} B_{j}\left|G\left(\omega=E_{B_{j}}+E_{B_{i}}\right)\right| B_{i} B_{j}\right\rangle,
$$

where the linear momentum of the intermediate singleparticle state $B_{j}$ is limited by the size of the Fermi surface $F_{j}$ for particle species $B_{j}$. The matrix element in Eq. (6) is properly antisymmetrized when the species $B_{i}$ and $B_{j}$ are the same. This equation is displayed in terms of Goldstone diagrams in Fig. 1. Diagram (a) represents contributions from nucleons only as hole states, while diagram (b) has only hyperons as holes states in case we have a finite hyperon fraction in $\beta$-stable neutron star matter. The external legs represent nucleons and hyperons. Detailed expressions for the single-particle energies and the $G$ matrices involved can be found in Ref. [9].

The total nonrelativistic energy density, $\varepsilon$, measured with respect to the nucleon mass, is obtained by adding the noninteracting leptonic contribution $\varepsilon_{l}$, and the baryonic contribution, $\varepsilon_{b}$, the latter being obtained from the baryon singleparticle potentials

$$
\begin{aligned}
\varepsilon=\varepsilon_{l}+\varepsilon_{b}= & \varepsilon_{l}+2 \sum_{B} \int_{0}^{k_{F}^{(B)}} \frac{d^{3} k}{(2 \pi)^{3}} \\
& \times\left(M_{B}+\frac{\hbar^{2} k^{2}}{2 M_{B}}+\frac{1}{2} U_{B}(k)-M_{N}\right) .
\end{aligned}
$$

The total binding energy per baryon $\mathcal{E}$ is then given by

$$
\mathcal{E}=\frac{\varepsilon}{\rho},
$$

where $\rho$ is the total baryonic density. The density of a given fermion species is given by 


$$
\rho_{i}=\frac{k_{F_{i}}^{3}}{3 \pi^{2}}=x_{i} \rho_{T}
$$

where $x_{i}$ is the fraction of particle species $i$ and $\rho_{T}=\rho+\rho_{l}$ is the total density which includes the baryonic $(\rho)$ and the leptonic one $\left(\rho_{l}\right)$.

In order to satisfy the equations for $\beta$-stable matter summarized in Eq. (3), we need to know the chemical potentials of the particles involved. In Brueckner-Hartree-Fock (BHF) theory the chemical potential is taken as the single particle energy at the Fermi momentum of the baryon $k_{F}^{(B)}$, which at the lowest order reads

$$
\mu_{B}=E_{B}\left(k_{F}^{(B)}\right)=M_{B}+T_{B}\left(k_{F}^{(B)}\right)+U_{B}^{N}\left(k_{F}^{(B)}\right)+U_{B}^{Y}\left(k_{F}^{(B)}\right),
$$

where, in the last equality, the baryon single-particle potential $U_{B}$ has been split into a contribution $U_{B}^{N}$, coming from the nucleonic Fermi seas $(p, n)$ and a contribution $U_{B}^{Y}$, coming from the hyperonic ones $\left(\Sigma^{-}, \Lambda, \ldots\right)$. From calculations in pure nucleonic matter it is well known that the nucleon chemical potential obtained from of Eq. (10) differs considerably from the thermodynamic definition

$$
\mu_{B}=\frac{\partial \varepsilon}{\partial \rho_{B}}
$$

Therefore, for the nucleons, we replace the nucleonic contribution to the chemical potential in Eq. (10), i.e., $\mu_{N}^{N}=M_{N}$ $+T_{N}\left(k_{F}^{(N)}\right)+U_{N}^{N}\left(k_{F}^{(N)}\right)$, by $\mu_{N}^{N}=\partial \varepsilon_{N N} / \partial \rho_{N}$, where

$$
\varepsilon_{N N}=2 \sum_{B=n, p} \int_{0}^{k_{F}^{(B)}} \frac{d^{3} k}{(2 \pi)^{3}}\left(M_{B}+\frac{\hbar^{2} k^{2}}{2 M_{B}}+\frac{1}{2} U_{B}^{N}(k)\right)
$$

is the nucleonic contribution to the baryonic energy density including only the interaction between $N N$ pairs. For the hyperons, we keep the prescription of Eq. (10). As shown in Ref. [7], these approximations amount to ignoring the weak dependence of $U_{N}^{N}, U_{Y}^{N}$ on the hyperon fractions and of $U_{N}^{Y}, U_{Y}^{Y}$ on the nucleon ones, and are good enough as long as the proton and hyperon fractions keep moderately small. Using the parabolic approximation for $\varepsilon_{N N}$ one obtains [7]

$$
\begin{aligned}
\mu_{p, n}\left(\rho_{N}, \beta\right)= & \mu_{p, n}\left(\rho_{N}, \beta=0\right) \\
& -\left(\beta^{2} \pm 2 \beta-\beta^{2} \rho_{N} \frac{\partial}{\partial \rho_{N}}\right) E_{\mathrm{sym}}\left(\rho_{N}\right),
\end{aligned}
$$

where $\beta=1-2 \rho_{p} / \rho_{N}$ is the asymmetry parameter, with $\rho_{N}$ $=\rho_{n}+\rho_{p}$. The symmetry energy $E_{\text {sym }}$ can be expressed as the difference of the energy per nucleon $\widetilde{\mathcal{E}}$ between pure neutron $(\beta=1)$ and symmetric nuclear $(\beta=0)$ matter:

$$
E_{\mathrm{sym}}\left(\rho_{N}\right)=\widetilde{\mathcal{E}}\left(\rho_{N}, \beta=1\right)-\widetilde{\mathcal{E}}\left(\rho_{N}, \beta=0\right)=\frac{1}{2} \frac{\partial \widetilde{\mathcal{E}}}{\partial \beta}\left(\rho_{N}, \beta=1\right),
$$

where $\widetilde{\mathcal{E}}$ is the nucleonic contribution to the total energy per nucleon, $\widetilde{\mathcal{E}}=\varepsilon_{N N} / \rho_{N}$, and $\mu_{p, n}\left(\rho_{N}, \beta=0\right)$ is given by

$$
\mu_{p, n}\left(\rho_{N}, \beta=0\right)=\widetilde{\mathcal{E}}\left(\rho_{N}, \beta=0\right)+\rho_{N} \frac{\partial \widetilde{\mathcal{E}}\left(\rho_{N}, \beta=0\right)}{\partial \rho_{N}}
$$

The many-body approach outlined above is the lowestorder BHF method extended to the hyperon sector. This means also that we consider only two-body interactions. However, it is well known from studies of nuclear matter and neutron star matter with nucleonic degrees of freedom only that three-body forces are important in order to reproduce the saturation properties of nuclear matter, see, e.g., Ref. [12] for the most recent approach. The effect of nucleon three-body forces on the properties of $\beta$-stable matter with hyperons has been studied in Refs. [6,7]. It is found that the repulsion induced by the three-body force at high densities enhances substantially the hyperon population and produces a strong softening of the EOS. In order to include such effects, we will alternatively use for the nucleonic sector, the EOS of Ref. [12] (hereafter referred to as APR98), which is obtained from a variational calculation using the Argonne $V_{18}$ nucleon-nucleon interaction [13] with relativistic boost corrections and a fitted three-body interaction model.

In the discussions below we will thus present two sets of results for $\beta$-stable matter, one where the nucleonic contributions to the self-energy of nucleons are derived from the baryon-baryon potential model of Stoks and Rijken [8] and one where the nucleonic contributions to the neutron and proton chemical potentials are calculated from the parametrization of the APR98 EOS discussed in Eq. (49) of Ref. [14]. We note that replacing the nucleon-nucleon part of the interaction model of Ref. [8] with that from the $V_{18}$ nucleonnucleon interaction [13], does not introduce large differences at the BHF level. However, the inclusion of three-body forces as done in Ref. [12] is important. Hyperonic contributions will, however, all be calculated with the baryon-baryon interaction of Stoks and Rijken [8]. We emphasize that, in the present work, $Y N$ as well as $Y Y$ interactions are taken into account.

\section{RESULTS}

\section{A. Equation of state and composition of $\boldsymbol{\beta}$-stable matter}

The above models for the pure nucleonic part (NSC97E and APR98) combined with the hyperon contribution (NSC97E) yield the composition of $\beta$-stable matter, up to total baryonic density $\rho=1.2 \mathrm{fm}^{-3}$, shown in Fig. 2. In the upper panel, results for the NSC97E model for $N N, Y N$, and $Y Y$ interactions are presented. Results combining the APR98 model for the nucleonic sector with the NSC97E for the $Y N$ and $Y Y$ interactions are shown in the lower panel. In both panels, solid lines correspond to the case in which all the interactions $N N, Y N$, and $Y Y$ are considered.

As can be seen by comparing the solid lines in both panels in Fig. 2, the composition of $\beta$-stable neutron star matter has a strong dependence on the model used to describe the 


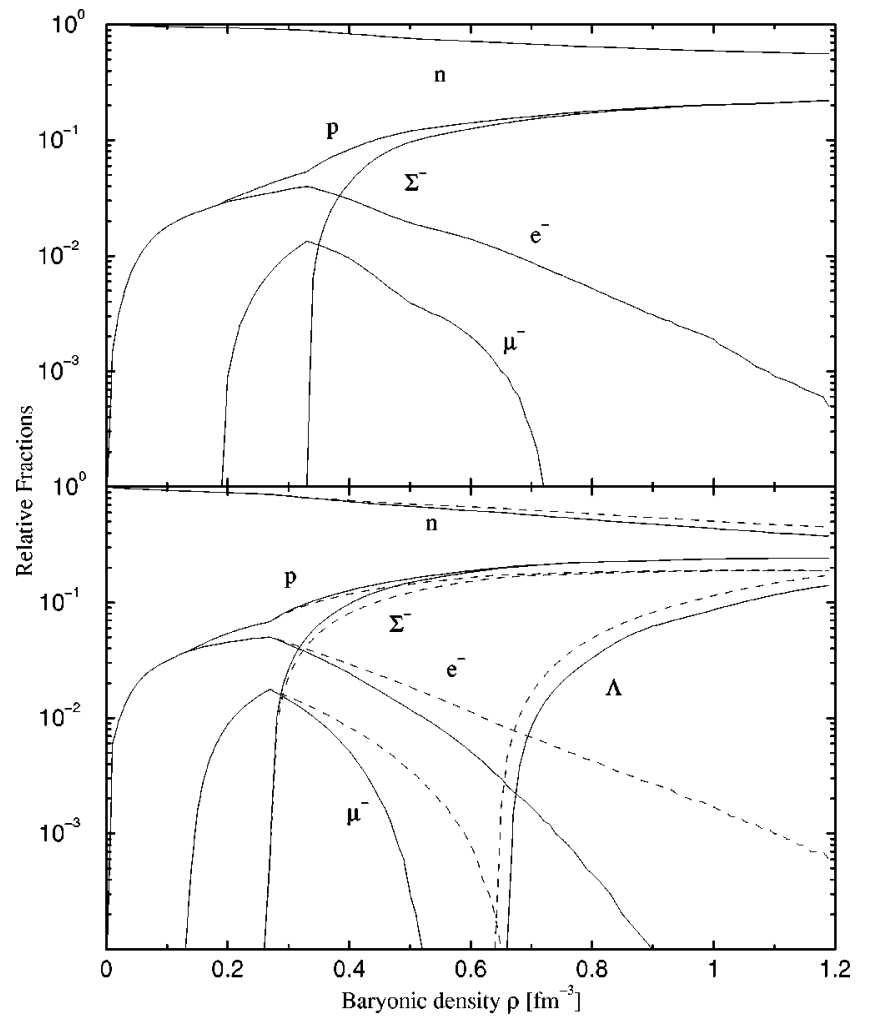

FIG. 2. Composition of $\beta$-stable neutron star matter. In the upper panel results for the Stoks and Rijken potential [8] are presented. In the lower panel the nucleonic part of the self-energy of the nucleons has been replaced with the EOS of Ref. [12]. Solid lines in upper and lower panel correspond to the case in which all the interactions (nucleon-nucleon, hyperon-nucleon, and hyperonhyperon) are considered. Dashed lines in the lower panel correspond to the case where the hyperon-hyperon interaction has been switched off.

nonstrange sector. In both cases, $\Sigma^{-}$is the first hyperon to appear due to its negative charge. Since the APR98 EOS yields a stiffer pure nucleonic matter EOS than the corresponding one for NSC97E, the onset of $\Sigma^{-}$for the APR98 case occurs at a smaller density $\left(\rho=0.27 \mathrm{fm}^{-3}\right)$ than for the NSC97E case $\left(\rho=0.34 \mathrm{fm}^{-3}\right)$. In both cases, as soon as the $\Sigma^{-}$hyperon appears, leptons tend to disappear, totally in the APR98 case (the electron chemical potential changes sign at $\rho=1.01 \mathrm{fm}^{-3}$, signaling the appearance of positrons), whereas in the NSC97E case only muons disappear. The onset of $\Lambda$ formation takes place at higher density. Recalling the condition for the appearance of $\Lambda, \mu_{\Lambda}=\mu_{n}=\mu_{p}+\mu_{e^{-}}$, and that the APR98 EOS is stiffer due to the inclusion of threebody forces, this clearly enhances the possibility of creating $\Lambda$ hyperons at lower density with this interaction model with respect to the NSC97E case. Indeed, the APR98 model produces $\Lambda$ hyperons from $\rho=0.67 \mathrm{fm}^{-3}$ on, whereas the neutron chemical potential of the NSC97E model turns out to be too small to equal the $\Lambda$ one in the range of densities explored. The absence of $\Lambda$ hyperons in the NSC97E case can also, in addition to a softer EOS, be retraced to a delicate balance between the nucleonic and hyperonic hole state contributions (and thereby to features of the baryon-baryon in-

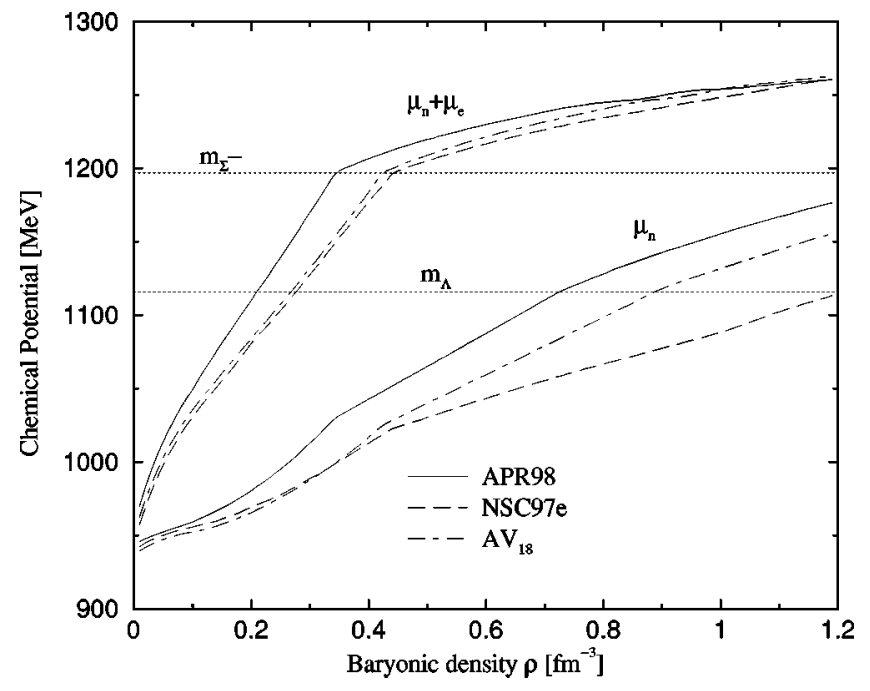

FIG. 3. Chemical equilibrium condition for the appearance of $\Sigma^{-}$and $\Lambda$ hyperons for the case of free hyperons and three different nucleon-nucleon interactions. Dotted straight lines denote the rest masses of the hyperons.

teraction) to the self-energy of the baryons considered here, see diagrams (a) and (b) in Fig. 1. Stated differently, the contributions from $\Sigma^{-}$, proton and neutron hole states to the $\Lambda$ chemical potential are not attractive enough to lower the chemical potential of the $\Lambda$ so that it equals that of the neutron. Furthermore, the increase of the chemical potential of the neutron with density is slowed down with the NSC97E $Y N$ interaction model since contributions from $\Sigma^{-}$hole states to the neutron self-energy are attractive, see, e.g., Ref. [9] for a detailed account of these aspects of the interaction model. We note that the isospin-dependent component (Lane term) of the $\Sigma^{-}$single-particle potential for the new Nijmegen interaction [8] is strongly attractive, as opposed to what is found $[15,17]$ for other interactions, including the old Nijmegen one [16]. This in turn implies a strong attraction for $\Sigma^{-} n(T=3 / 2)$ pairs, which is 10 times that obtained for the old Nijmegen potential at saturation density. These differences become more noticeable as density increases: while the $\Sigma^{-} n$ pairs become increasingly more attractive with the new Nijmegen potentials (see, e.g., Fig. 5 in Ref. [9]), they turn out to be strongly repulsive for the old one (see, e.g., Figs. 1 and 2 in Ref. [7]). This is why in Ref. [7] the onset density for the appearance of $\Sigma^{-}$is larger than that for free hyperons, whereas the reverse situation is found here (compare the $\Sigma^{-}$onset point in Fig. 2 with what would be extracted from Fig. 3). Within our many-body approach, no other hyperons appear at densities below $\rho=1.2 \mathrm{fm}^{-3}$. These results differ from present mean field calculations [1-3], where all kinds of hyperons can appear at the densities considered here. Although the APR98 EOS may be viewed as the currently most realistic approach to the nucleonic EOS, our results have to be gauged with the uncertainty in the hyperon-hyperon and hyperon-nucleon interactions. Especially, if the hyperon-hyperon interactions tend to be more attractive, this may lead to the formation of hyperons such as the $\Lambda, \Sigma^{0}, \Sigma^{+}, \Xi^{-}$, and $\Xi^{0}$ at lower densities. The stiffness of the nucleonic contribution, together with the 
hyperon-nucleon and hyperon-hyperon interactions play crucial roles in the appearance of various hyperons beyond the $\Sigma^{-}$.

In order to examine the role of the hyperon-hyperon interaction on the composition of $\beta$-stable neutron star matter, the dashed lines in the lower panel of Fig. 2 show the results of a calculation in which only the $N N$ and $Y N$ interactions are taken into account. When the $Y Y$ interaction is switched off, the scenario described above changes only quantitatively. The onset point of $\Sigma^{-}$does not change, because $\Sigma^{-}$ is the first hyperon to appear and therefore the $Y Y$ interaction plays no role for densities below this point. We note that the reduction of the $\Sigma^{-}$fraction compared with the case which includes the $Y Y$ interaction, is a consequence of neglecting the strongly attractive $\Sigma^{-} \Sigma^{-}$interaction [8], which allows the energy balance $\left(n n \leftrightarrow \Sigma^{-} p\right)$ to be fulfilled with a smaller $\Sigma^{-}$Fermi sea. In turn, the reduction of the $\Sigma^{-}$fraction yields a moderate increase of the leptonic content in order to keep charge neutrality (in fact only muons disappear now). On the other hand, a smaller amount of $\Sigma^{-}$'s implies less $\Sigma^{-} n$ pairs. Recalling that the $\Sigma^{-} n$ interaction is attractive in this model (see, e.g., Fig. 7 of Ref. [9]), this means that the chemical potential of the neutrons now becomes less attractive. As a consequence, the $\Lambda$ hyperons appear at a smaller density $\left(\rho=0.65 \mathrm{fm}^{-3}\right)$ and have a larger relative fraction.

As it has been mentioned, the composition of $\beta$-stable matter depends strongly on the model used to describe the nucleonic sector. In order to study this dependence, Fig. 3 shows the chemical potential of the neutron and the sum $\mu_{n}+\mu_{e^{-}}$for $\beta$-stable matter composed of nucleons and free hyperons for three different $N N$ interaction models. The solid lines correspond to the variational calculation denoted by APR98, which uses the Argonne $V_{18}$ interaction and includes three-body forces and relativistic boost corrections. The dashed and dot-dashed lines correspond to lowest-order BHF calculations using, respectively, the NSC97E and the Argonne $V_{18}$ potentials, the latter extracted from the results of Ref. [7]. Dotted lines denote the $\Lambda$ and $\Sigma^{-}$masses. In this case, the onset conditions for $\Lambda$ and $\Sigma^{-}$are, respectively, $\mu_{n}=m_{\Lambda}$ and $\mu_{n}+\mu_{e^{-}}=m_{\Sigma^{-}}$. As can bee seen from the figure, the onset points of both hyperons are different depending on the $N N$ interaction model employed. In the APR98 model both the $\Sigma^{-}$and $\Lambda$ hyperons appear at lower densities than in the lowest-order BHF models using the Argonne $V_{18}$ or the NSC97E interactions. This is a consequence of the different stiffness of the EOS generated by the three $N N$ interaction models. In fact, the NSC97E interaction gives the softest EOS and it is not even able to produce $\Lambda$ hyperons in the range of densities explored. Note that the hyperon onset points determined from Fig. 3 differ slightly from those observed in Fig. 2 as a consequence of the effect of the $Y N$ and $Y Y$ interactions. Since the differences are not so large one concludes that the main features of the composition of matter are dominated by the pure nucleonic contribution to the EOS.

At present, the APR98 EOS represents perhaps the most sophisticated many-body approach to nuclear matter. Therefore, in what follows, we restrict our results to this $N N$ in-

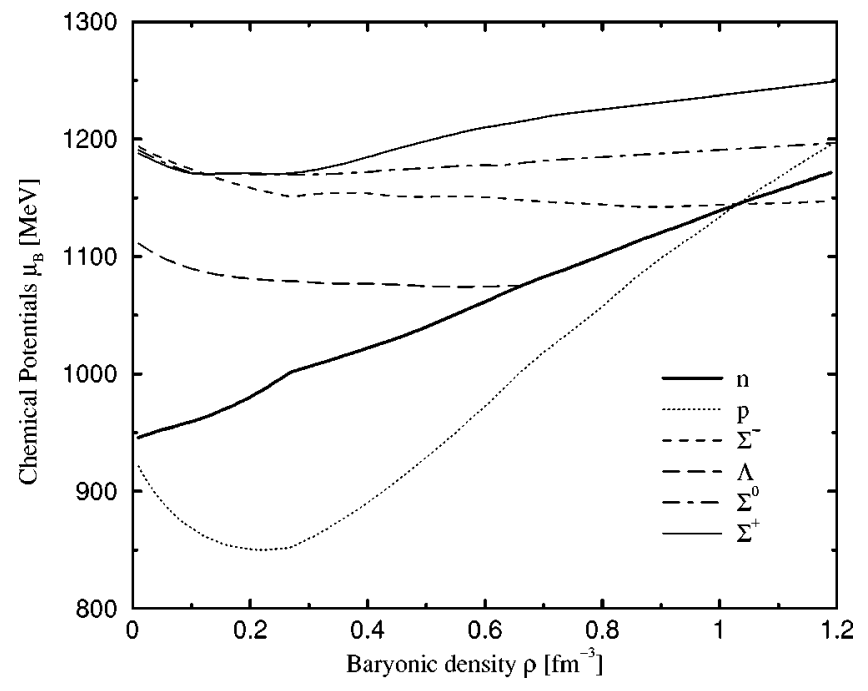

FIG. 4. Chemical potentials in $\beta$-stable neutron star matter as functions of the total baryonic density $\rho$.

teraction model supplemented with the NSC97E one for the hyperonic sector.

In Fig. 4 we show the chemical potentials in $\beta$-stable neutron star matter for different baryons. We note that neither the $\Sigma^{0}$ nor the $\Sigma^{+}$do appear since, as seen from the figure, the respective stability criteria of Eq. (3) are not fulfilled. This is due, partly, to the fact that none of the $\Sigma^{0}$-baryon and the $\Sigma^{+}$-baryon self-energies are attractive enough. A similar argument applies to $\Xi^{0}$ and $\Xi^{-}$. In the latter case the mass of the particle is $\sim 1315 \mathrm{MeV}$ and an attraction of around $200 \mathrm{MeV}$ would be needed to fulfill the condition $\mu_{\Lambda}=\mu_{\Xi^{0}}=\mu_{n}$ at the highest density explored in this work. From the figure we see, however, that the $\Sigma^{0}$ hyperon could appear at densities close to $1.3 \mathrm{fm}^{-3}$.

Figure 5 shows the EOS for four different cases: pure nucleonic matter (solid line), matter with nucleons and free hyperons (dotted line), matter with nucleons and hyperons interacting only with nucleons (dashed line), and finally, matter with nucleons and hyperons interacting both with nucleons and hyperons (long-dashed line). Each curve corresponds to a different composition of $\beta$-stable neutron star matter, obtained by requiring the equilibrium conditions of Eq. (3), with the appropriate chemical potentials for each of the four cases. The leptonic contribution to the EOS is also included in all the cases. As can be seen comparing the solid and dotted lines the appearance of hyperons leads to a considerable softening of the EOS. This softening is essentially due to a decrease of the kinetic energy because the hyperons can be accommodated in lower momentum states and in addition have a large bare mass. The hyperon-nucleon interaction (dashed line) has two effects. On one hand, for densities up to $\rho \sim 0.72 \mathrm{fm}^{-3}$, the $Y N$ interaction reduces the total energy per baryon making the EOS even softer. On the other hand, for densities higher than $\rho=0.72 \mathrm{fm}^{-3}$, it becomes repulsive and therefore, the EOS becomes slightly stiffer than that for noninteracting hyperons. The contribution from the hyperon-hyperon interaction (long-dashed line) is always attractive producing a softening of the EOS over the whole range of densities explored. We note that, at high densities, 


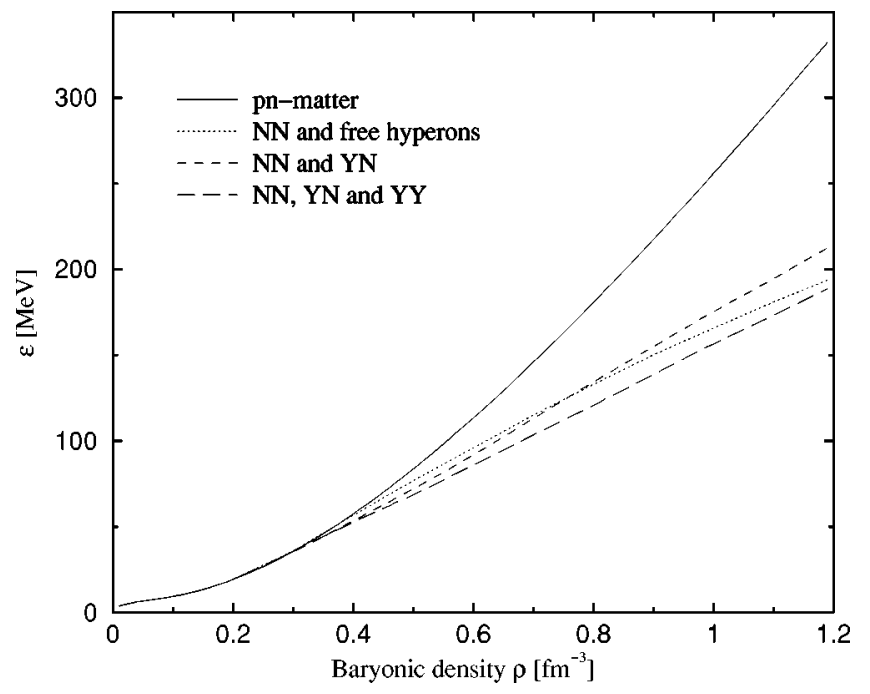

FIG. 5. Energy per baryon in $\beta$-stable neutron star matter as a function of the total baryonic density $\rho$, for four cases: pure nucleonic matter (solid lines); matter with nucleons and noninteracting hyperons (dotted line); matter with nucleons and hyperons interacting only with nucleons (dashed line); and matter with nucleons and hyperons interacting with nucleons and hyperons (long-dashed line). The leptonic contribution to the energy per baryon is included in all cases.

the combined incorporation of the repulsive $Y N$ and attractive $Y Y$ interactions causes the EOS to become closer to that for noninteracting hyperons.

\section{B. Structure of neutron stars}

We end this section with a discussion on neutron star properties with the above equations of state. The best determined neutron star masses are found in binary pulsars and all lie in the range $1.35 \pm 0.04 M_{\odot}[18]$ except for the nonrelativistic pulsar PSR $\mathrm{J} 1012+5307$ of mass $M=(2.1$ $\pm 0.8) M_{\odot}[19]$. Several x-ray binary masses have been measured of which the heaviest are Vela X-1 with $M=(1.9$ $\pm 0.2) M_{\odot}[20]$ and Cygnus X-2 with $M=(1.78 \pm 0.2) M_{\odot}$ [21]. The recent discovery of high-frequency brightness oscillations in low-mass $\mathrm{x}$-ray binaries provides a promising new method for determining masses and radii of neutron stars, see Ref. [22]. The kilohertz quasiperiodic oscillations (QPO) occur in pairs and are most likely the orbital frequencies of accreting matter in Keplerian orbits around neutron stars of mass $M$ and its beat frequency with the neutron star spin. According to Zhang et al. [23] and Kaaret et al. [24] the accretion can for a few QPO's be tracked to its innermost stable orbit. For slowly rotating stars the resulting mass is $M \simeq 2.2 M_{\odot}\left(\mathrm{kHz} / \nu_{\mathrm{QPO}}\right)$. For example, the maximum frequency of $1060 \mathrm{~Hz}$ upper QPO observed in 4U 1820-30 gives $M \simeq 2.25 M_{\odot}$ after correcting for the neutron star rotation frequency. If the maximum QPO frequencies of $4 \mathrm{U}$ $1608-52\left(\nu_{\mathrm{QPO}}=1125 \mathrm{~Hz}\right)$ and $4 \mathrm{U}$ 1636-536 $\left(\nu_{\mathrm{QPO}}=1228\right.$ $\mathrm{Hz}$ ) also correspond to innermost stable orbits the corresponding masses are $2.1 M_{\odot}$ and $1.9 M_{\odot}$. These observations define a range of variation for the mass between $M$ $\sim 1.35 M_{\odot}$ and $M \sim 2.2 M_{\odot}$, which severely restricts the EOS for dense matter.

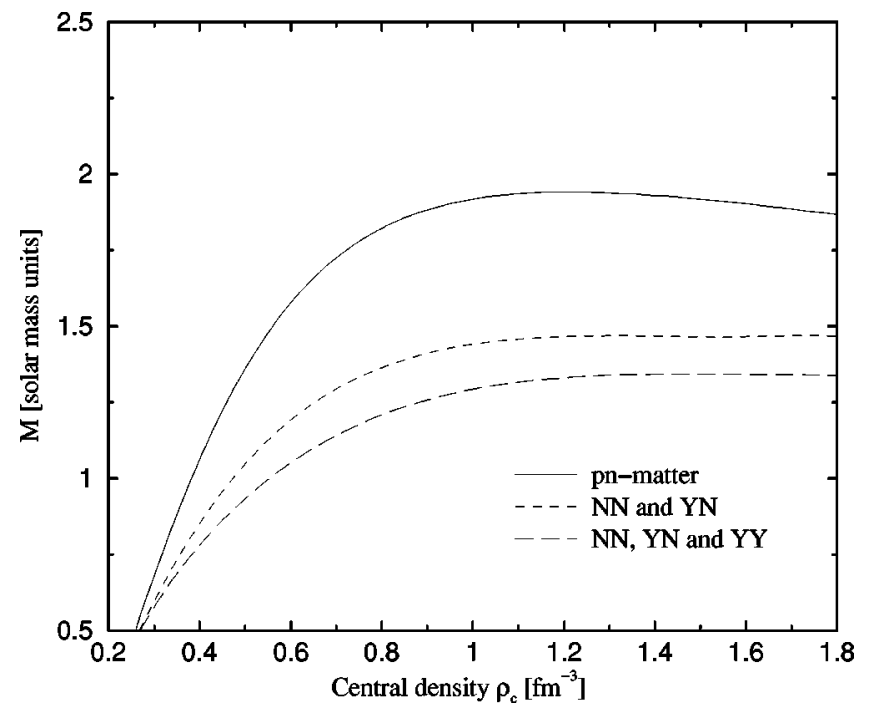

FIG. 6. Total mass $M$ for various equations of state. Rotational corrections are not included. The solid line corresponds to the case of $\beta$-stable matter with nucleonic degrees of freedom only, the short-dashed line includes also the effects of the hyperon-nucleon interaction, while the long-dashed line includes the three types of baryon-baryon interactions.

In order to obtain the radius and mass of a neutron star, we have solved the Tolman-Oppenheimer-Volkov (TOV) equation with and without rotational corrections. The latter corrections imply an increase of mass to balance the rotational energy. Accounting for rotations leads to corrections to the TOV equation and the total mass that include also deformations from spherical symmetry. We assume that such contributions from deformations are negligible for a slowly rotating star, resulting in modified equations for the pressure and the mass as shown in the work of Hartle [25], or as detailed in Eqs. (123) and (124) of Ref. [14].

Following the discussion of Figs. 3, 4, and 5, we use the pure nucleonic matter EOS of Akmal et al. [12], including both relativistic boost corrections and three-body interactions. Relating to the discussion of the previous subsection, we study two additional cases as well. One case where we just include the hyperon-nucleon interaction as done in, e.g., Ref. [7] and another case where we include both the hyperon-nucleon and the hyperon-hyperon interactions.

In Fig. 6 we show the resulting mass as function of these equations of state without rotational corrections. In Fig. 7 we include rotational corrections to the mass. The mass-radius relations for the same equations of state are shown in Fig. 8.

For $\beta$-stable pure nucleonic matter the EOS is rather stiff compared with the EOS obtained with hyperons, see Fig. 5. The EOS yields a maximum mass $M=1.89 M_{\odot}$ without rotational corrections and $M=2.11 M_{\odot}$ when rotational corrections are included. The results for the masses as functions of central density $\rho_{c}$ are shown by the solid lines in Figs. 6 and 7. The corresponding mass-radius relation for masses without rotational corrections are shown in Fig. 8.

The second set of results refers to the case where we allow for the presence of hyperons and consider the hyperonnucleon interaction but explicitly exclude the hyperon- 


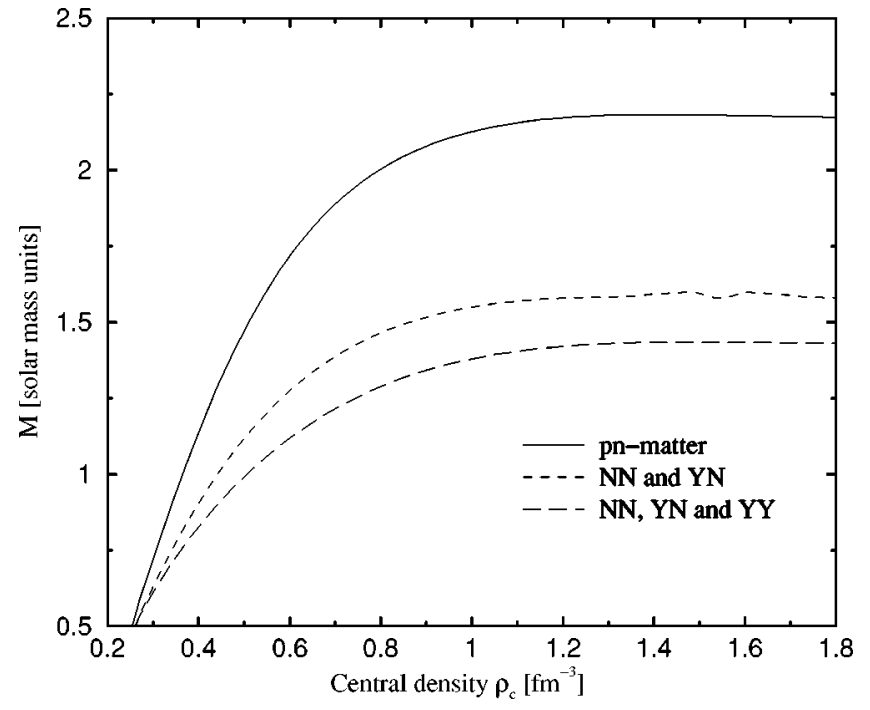

FIG. 7. Same as previous figure, but with rotational corrections.

hyperon one. The corresponding results are shown by the short-dashed lines in Figs. 6, 7, and 8. Without rotational corrections, we obtain a maximum mass $M=1.47 M_{\odot}$ whereas the rotational correction increases the mass to $M$ $=1.60 M_{\odot}$. Thus, the inclusion of the the $Y N$ interaction with the corresponding formation of $\Sigma^{-}$and $\Lambda$ leads to a reduction of the mass by $\sim 0.4-0.5 M_{\odot}$. This large reduction is mainly a consequence of the strong softening of the EOS due to appearance of hyperons.

The last EOS employed is that which combines the nucleonic part of Ref. [12] with the computed hyperon contribution including both the hyperon-nucleon and the hyperonhyperon interactions. These results are shown by the longdashed lines in Figs. 6, 7, and 8. The inclusion of the hyperon-hyperon interaction leads to a further softening of the EOS in Fig. 5, and, as can be seen from Fig. 6, this leads to an additional reduction of the total mass. Without rotational corrections we obtain a maximum mass $M$

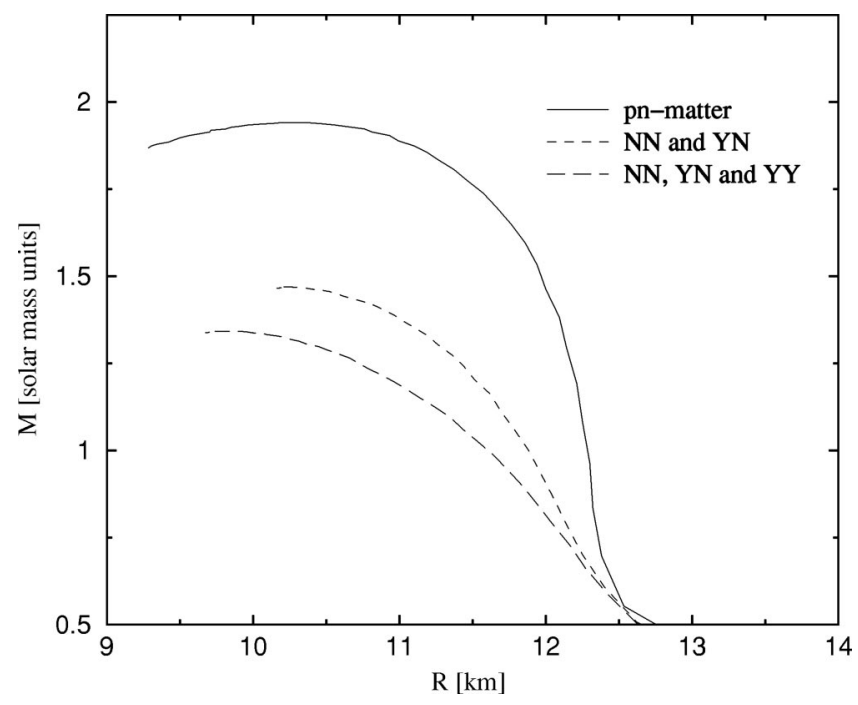

FIG. 8. Mass-radius relation without rotational corrections. Notations as in the previous two figures. $\sim 1.34 M_{\odot}$, while the rotational correction increases the mass to $M \sim 1.44 M_{\odot}$. The size of the reduction due to the presence of hyperons $\Delta M \sim 0.6-0.7 M_{\odot}$, and the obtained neutron star masses are comparable to those reported by Balberg et al. [5].

There are other features as well to be noted from Figs. 6, 7 , and 8 . The EOS with hyperons reaches a maximum mass at a central density $\rho_{c} \sim 1.3-1.4 \mathrm{fm}^{-3}$. In Fig. 2 we showed that the only hyperons which can appear at these densities are $\Lambda$ and $\Sigma^{-}$. If other hyperons were to appear at higher densities, this would most likely lead to a further softening of the EOS, and thereby smaller neutron star masses.

The reader should, however, note that our calculation of hyperon degrees freedom is based on a nonrelativistic BHF approach. Although the nucleonic part extracted from Ref. [12], including three-body forces and relativistic boost corrections, is to be considered as a benchmark calculation for nucleonic degrees of freedom, relativistic effects in the hyperonic calculation could result in a stiffer EOS and thereby larger mass. However, relativistic mean field calculations with parameters which result in a similar composition of matter as shown in Fig. 2, result in similar masses as those reported in Fig. 6. In this sense, our results with and without hyperons may provide a lower and upper bound for the maximum mass. This leaves two natural options when compared to the observed neutron star masses. If the above heavy neutron stars prove erroneous by more detailed observations and only masses similar to those of binary pulsars are found, this may indicate that heavier neutron stars simply are not stable which in turn implies a soft EOS, or that a significant phase transition must occur already at a few times nuclear saturation densities. Our EOS with hyperons would fit into this case, although the mass without rotational corrections is on the lower side. If the large masses from QPO's are confirmed, then the EOS for baryonic matter needs to be stiffer. This would then pose a severe problem to present hadronic models since, when the nucleonic part of the EOS is sufficiently stiff to support large masses, one cannot avoid the appearance of hyperons which, in turn, produce a softening of the EOS.

Although we have only considered the formation of hyperons in neutron stars, transitions to other degrees of freedom such as quark matter, kaon condensation, and pion condensation may or may not take place in neutron star matter. We would, however, like to emphasize that the hyperon formation mechanism is perhaps the most robust one and is likely to occur in the interior of a neutron star, unless the hyperon self-energies are strongly repulsive due to repulsive hyperon-nucleon and hyperon-hyperon interactions, a repulsion which would contradict present data on hypernuclei [26]. The EOS with hyperons yields, however, neutron star masses without rotational corrections which are even below $\sim 1.4 M_{\odot}$. This means that our EOS with hyperons needs to be stiffer, a fact which may in turn imply that more complicated many-body terms not included in our calculations, such as three-body forces between nucleons and hyperons and/or relativistic effects, are needed. 


\section{CONCLUSIONS}

Employing the recent parametrization of the free baryonbaryon potentials for the complete baryon octet of Stoks and Rijken [8], we have performed a microscopic many-body calculation of the structure of $\beta$-stable neutron star matter including hyperonic degrees of freedom. The potential model employed allows for the presence of only two types of hyperons up to densities of about ten times nuclear matter saturation density. These hyperons are $\Sigma^{-}$and $\Lambda$. The interactions for strangeness $S=-1, S=-2, S=-3$, and $S=-4$ are not attractive enough to allow the formation of other hyperons.

The presence of hyperons leads, however, to a considerable softening of the EOS, entailing a corresponding reduction of the maximum mass of the neutron star. With hyperons, including only the hyperon-nucleon interaction, we obtain maximum masses of the order $M=1.47 M_{\odot}$ and $M$ $=1.60 M_{\odot}$ without and with rotational corrections, respectively. The inclusion of the hyperon-hyperon interaction leads to a further softening of the EOS and reduces the obtained masses to $M=1.34 M_{\odot}$ and $M=1.44 M_{\odot}$ without and with rotational corrections, respectively. The corresponding numbers with just nucleonic degrees of freedom are $M$ $=1.89 M_{\odot}$ and $M=2.11 M_{\odot}$, showing that the reduction in mass due to hyperonic degrees of freedom is $\sim 0.5$ $-0.7 M_{\odot}$.

Our novel result is that a further softening of the EOS is obtained when including the effect of the $Y Y$ interaction since it is attractive over the whole density range explored. This is mainly due to the $\Sigma \Sigma$ interaction which is strongly enough to develop a bound state [8]. We note that the $\Lambda \Lambda$ attraction produced by this model is only mild, not being able to reproduce the experimental $2 \Lambda$ separation energy of $\Delta B_{\Lambda \Lambda} \sim 4-5 \mathrm{MeV}$ [27].

Whether this additional softening is realistic or not will depend on the details of the $Y Y$ interaction that is, unfortunatelly, not well constrained at present. New data in the $S$ $=-2$ sector, either from double- $\Lambda$ hypernuclei or from $\Xi^{-}$-atoms, are very much awaited for.

\section{ACKNOWLEDGMENTS}

We are much indebted to M. Baldo, F. Burgio, $\varnothing$. Elgarøy, H. Heiselberg, H.-J. Schulze, and V. G. J. Stoks for many useful comments. This work has been supported by the DGICYT (Spain) Grant No. PB95-1249, the Program SCR98-11 from the Generalitat de Catalunya, and the Research Council of Norway. One of the authors (I.V.) wishes to acknowledge support from the Ministerio de Educación y Cultura (Spain).
[1] M. Prakash, I. Bombaci, M. Prakash, P. J. Ellis, J.M. Lattimer, and R. Knorren, Phys. Rep. 280, 1 (1997).

[2] R. Knorren, M. Prakash, and P. J. Ellis, Phys. Rev. C 52, 3470 (1995).

[3] J. Schaffner and I. Mishustin, Phys. Rev. C 53, 1416 (1996).

[4] S. Balberg and A. Gal, Nucl. Phys. A625, 435 (1997).

[5] S. Balberg, I. Lichtenstadt, and G. B. Cook, Astrophys. J. Suppl. Ser. 121, 515 (1999).

[6] H.-J. Schulze, M. Baldo, U. Lombardo, J. Cugnon, and A. Lejeune, Phys. Rev. C 57, 704 (1998); M. Baldo, G. F. Burgio, and H.-J. Schulze, ibid. 58, 3688 (1998).

[7] M. Baldo, G.F. Burgio, and H.-J. Schulze, Phys. Rev. C 61, 055801 (2000).

[8] V. G. J. Stoks and Th. A. Rijken, Phys. Rev. C 59, 3009 (1999).

[9] I. Vidaña, A. Polls, A. Ramos, M. Hjorth-Jensen, and V. G. J. Stoks, Phys. Rev. C 61, 025802 (2000).

[10] Th. A. Rijken, V. G. J. Stoks, and Y. Yamamoto, Phys. Rev. C 59, 21 (1998).

[11] V. G. J. Stoks and T.-S. H. Lee, Phys. Rev. C 60, 024006 (1999).

[12] A. Akmal, V. R. Pandharipande, and D. G. Ravenhall, Phys. Rev. C 58, 1804 (1998).

[13] R. B. Wiringa, V. G. J. Stoks, and R. Schiavilla, Phys. Rev. C 51, 38 (1995).

[14] H. Heiselberg and M. Hjorth-Jensen, Phys. Rep. 328, 237 (2000); Astrophys. J. Lett. 525, L41 (1999).
[15] I. Vidaña, A. Polls, A. Ramos, and M. Hjorth-Jensen, Nucl. Phys. A644, 201 (1998).

[16] P. M. M. Maessen, T. A. Rijken, and J. J. de Swart, Phys. Rev. C 40, 2226 (1989).

[17] J. Dabrowski, Phys. Rev. C 61, 025802 (1999).

[18] S. E. Thorsett and D. Chakrabarty, Astrophys. J. 512, 288 (1999).

[19] J. van Paradijs, astro-ph/9802177, in The Many Faces of Neutron Stars, edited by R. Buccheri, J. van Paradijs, and M.A. Alpar (Kluwer, Dordrecht, in press).

[20] S. Barziv et al. (unpublished).

[21] J.A. Orosz and E. Kuulkers, astro-ph/9901177; E. Kuulkers (private communication).

[22] M. C. Miller, F. K. Lamb, and P. Psaltis, Astrophys. J. 508, 791 (1998).

[23] W. Zhang, T. E. Strohmayer, and J. H. Swank, Astrophys. J. Lett. 482, L167 (1997); W. Zhang, A. P. Smale, T. E. Strohmayer, and J. H. Swank, ibid. 500, L171 (1998).

[24] P. Kaaret, E. C. Ford, and K. Chen, Astrophys. J. Lett. 480, L27 (1997).

[25] J. B. Hartle, Astrophys. J. 150, 1005 (1967).

[26] H. Bandō, T. Motoba, and J. Žofka, Int. J. Mod. Phys. A 5, 4021 (1990).

[27] D. J. Prowse, Phys. Rev. Lett. 17, 782 (1966); M. Danysz et al., Nucl. Phys. 49, 121 (1963); reanalyzed in R.H. Dalitz, Proc. R. Soc. London, Ser. A 426, 1 (1989); S. Aoki et al., Prog. Theor. Phys. 85, 1287 (1991); G. B. Franklin, Nucl. Phys. A585, 83c (1995). 